\title{
J-PET detector system for studies of the electron-positron annihilations
}

\author{
M. Pawlik-Niedźwiecka ${ }^{1}$, O. Khreptak ${ }^{1, \star}$, A. Gajos ${ }^{1}$, A. Wieczorek ${ }^{1,2}$, D. Alfs ${ }^{1}$, T. Bednarski ${ }^{1}$, \\ P. Białas ${ }^{1}$, C. Curceanu ${ }^{3}$, E. Czerwiński ${ }^{1}$, K. Dulski ${ }^{1}$, B. Głowacz ${ }^{1}$, N. Gupta-Sharma ${ }^{1}$, \\ M. Gorgol ${ }^{4}$, B. C. Hiesmayr ${ }^{5}$, B. Jasińska ${ }^{4}$, D. Kamińska ${ }^{1}$, G. Korcyl ${ }^{1}$, P. Kowalski ${ }^{6}$, W. Krzmieńn ${ }^{7}$, \\ N. Krawczyk ${ }^{1}$, E. Kubicz ${ }^{1}$, M. Mohammed ${ }^{1}$, Sz. Niedźwiecki ${ }^{1}$, L. Raczyński ${ }^{6}$, Z. Rudy ${ }^{1}$, \\ M. Silarski ${ }^{1}$, W. Wiślicki ${ }^{6}$, B. Zgardzińska ${ }^{4}$, M. Zieliński ${ }^{1}$, and P. Moskal ${ }^{1}$
}

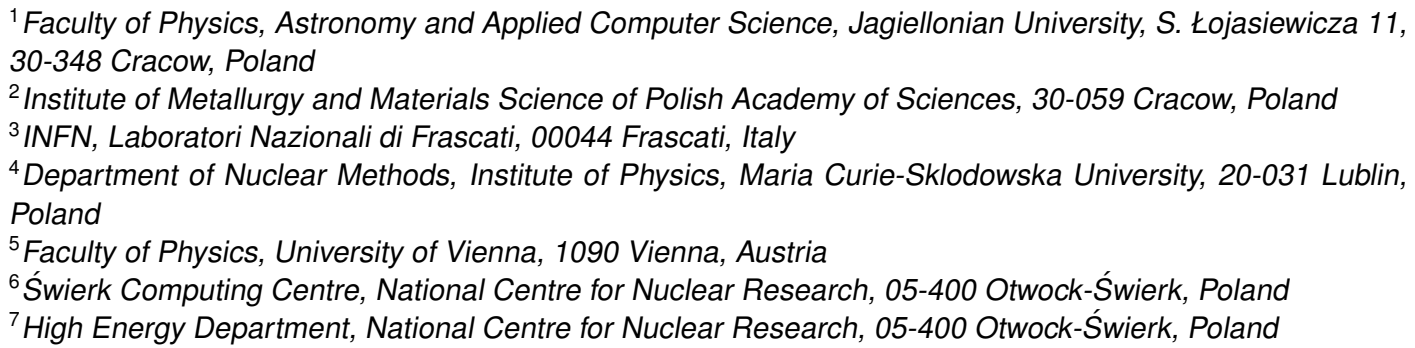

\begin{abstract}
Jagiellonian Positron Emission Tomograph (J-PET) has been recently constructed at the Jagiellonian University as a prototype of a cost-effective scanner for the metabolic imaging of the whole human body. J-PET detector is optimized for the measurement of momentum and polarization of photons from the electron-positron annihilations. It is built out of strips of plastic scintillators, forming three cylindrical layers. As detector of gamma quanta it will be used for studies of discrete symmetries and multiparticle entanglement of photons originating from the decays of ortho-positronium atoms.
\end{abstract}

\section{Introduction}

The Jagiellonian Positron Emission Tomograph (J-PET) has been constructed as a prototype of the novel cost-effective scanner for the metabolic imaging of the whole human body [1]. Using long strips of plastic scintillators as radiation detectors it has been optimized for the detection of photons from the electron-positron annihilation with high time- and high angular-resolution. J-PET as a multi-purpose detector provides new opportunities for studying decays of ortho-positronium atoms ( $o$-Ps). Studies of the three-gamma $o$-Ps decays allow for searches of discrete symmetries violation and physics beyond the Standard Model (e.g. extra dimensions, dark matter and a new light vector gauge boson) [1, 2]. Moreover, determination of the angles between $o$-Ps spin and the scattering planes of photons will

^e-mail: alexander.khreptak@gmail.com 
allow for the multi-particle entanglement studies of high energy photons originating from the positronium decay. In this article we briefly describe the concept of the J-PET detector and its potential in the studies of the fundamental properties of leptonic systems. Detailed research program is described in Ref. [1].

\section{Jagiellonian Positron Emission Tomograph (J-PET)}

A novel concept of PET scanner, referred to as Jagiellonian Positron Emission Tomograph, was developed at the Jagiellonian University in Cracow. In the J-PET detector, axially arranged organic scintillators strips form a barrel. Light signals, generated by gamma quanta interaction with scintillator, are read out by pairs of photomultipliers connected to the opposite ends of each strip. Scheme of two-strips J-PET setup is presented in Fig. 1.

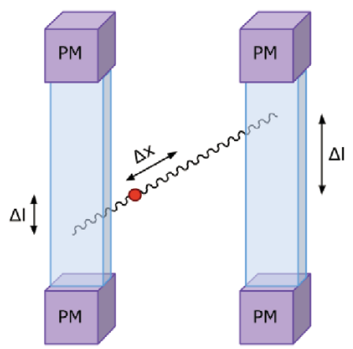

Figure 1. Scheme of the two-strips J-PET setup. Place of $511 \mathrm{keV} \gamma$ quanta interaction with scintillator material $(\Delta l)$ measured relative to the strip center is determined from the difference between light signals arrival times to both photomultipliers. Place of the two-photon annihilation event $(\Delta x)$, along the line of response (LOR), is defined by time difference between two modules as described in detail in references [3-5].

First prototype of the multi-purpose J-PET scanner consists of three layers of EJ-230 scintillators strips read-out by Hamamatsu R9800 photomultipliers and forming a cylinder of diameter equal to $85 \mathrm{~cm}$ and its active inner part is equal to $50 \mathrm{~cm}$, as one can see in right panel of Fig. 2. Scintillators are wrapped with Vikuiti foil and additionally covered with light-tight black foil. Electrical signals from each photomultiplier are probed by multi-threshold digital electronics with accuracy equal to 30 ps [4].
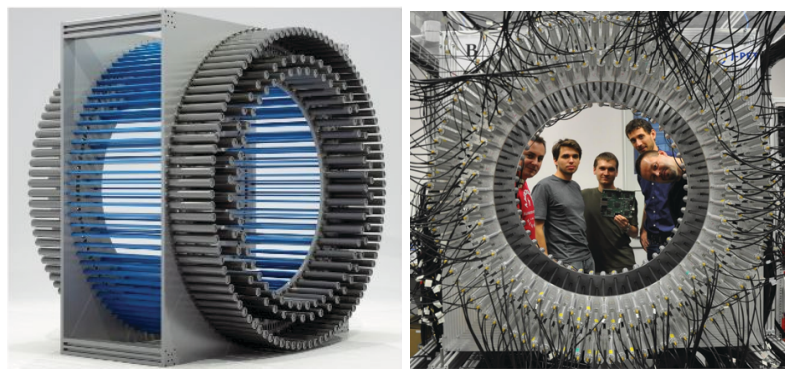

Figure 2. (Left) Visualization of the J-PET detector prototype. One can see three layers of scintillator strips connected with photomultipliers and arranged in a barrel. (Right) Photograph of the first prototype of the J-PET detector together with few members of the collaboration.

Data from electronics is collected by trigger-less acquisition system. Both the dedicated front-end electronics, as well as the acquisition system, were designed by the J-PET collaboration [6]. New methods for reconstruction of position and time of interaction of $\gamma$ quanta based on compressive sensing theory and library of model signals has been also developed [7, 8]. The J-PET detector is 
optimized for the detection of gamma quanta originating from the electron-positron annihilation, but it can be also used for studies of ortho-positronium decays, as well as studies of discrete symmetries, which are briefly described in the next section.

\section{Discrete symmetries in decays of positronium atoms}

Positronium is leptonic object build out of electron and anti-electron (positron), so it is an eigenstate of charge (C) and spatial (P) parity, as well as their combination (CP) [9]. Therefore, it is an unique to test discrete symmetries with precision limited in principle only by the weak and photon-photon interactions [1]. The search for a possible violation of these symmetries may be done by measurement of the expectation values of various operators, odd with respect to the studied symmetry, constructed from momenta of photons and the spin of the ortho-positronium [1]. Polarized pozitronium is produced in the layer of porous material (eg. aerogel or polymer [10]) surrounding radioactive source providing longitudinally polarized positrons in a parity violating beta-plus decay (eg. sodium ${ }^{22} \mathrm{Na}$ ). Positronium created this way decays into three photons which can interact via Compton effect with scintillator material. Position of the positronium annihilation can be determined based on time and positions of interaction of photons with scintillator using trilateration-based reconstruction [11]. Registration of de-excitation photon from the excited daughter nucleus created in the beta-plus decay enables also estimation of positronium lifetime.

\section{Summary}

J-PET being a novel solution in the Positron Emmision Tomography provides also new opportunities to study fundamental physics in the leptonic sector $[1,2,11,12]$ as well as in the life- and materialsciences $[5,13-15]$. In particular it has a potential for improvement of present limits $[16,17]$ of the decays of positronium [1].

\section{References}

[1] P. Moskal et al., Acta Phys. Polon. B 47, 509 (2016)

[2] D. Kamińska et al., Eur. Phys. J. C 76, 445 (2016)

[3] P. Moskal et al., Nucl. Instr. Meth. Phys. Res. A 775, 54 (2015)

[4] P. Moskal et al., Acta Phys. Pol. B 47, 509 (2016)

[5] P. Moskal et al., Phys. Med. Biol. 61, 2025 (2016)

[6] G. Korcyl et al., Acta Phys. Pol. B 47, 491 (2016)

[7] L. Raczyński et al., Nucl. Instrum. Meth. A 786, 105 (2015)

[8] N. G. Sharma et al., Nukleonika 60, 765 (2015)

[9] M. Deutsch, Phys. Rev. 82, 455 (1951)

[10] B. Jasińska et al., Acta Phys. Pol. B 47, 453 (2016)

[11] A. Gajos et al., Nucl. Instrum. Meth. A 819, 54 (2016)

[12] D. Kamińska et al., Nukleonika 60, 729 (2015)

[13] E. Kubicz et al., Nukleonika 60, 749 (2015)

[14] A. Wieczorek et al., Acta Phys. Pol. A 127, 1487 (2015)

[15] A. Wieczorek et al., Nukleonika 60, 777 (2015)

[16] T. Yamazaki et al., Phys. Rev. Lett. 104, 083401 (2010)

[17] P. A. Vetter, S. J. Freedman, Phys. Rev. Lett. 91, 263401 (2003) 